\title{
Evaluating Tactile Sensitivity Adaptation by Measuring the Differential Threshold of Archers
}

\author{
Kentaro Kotani $^{1)}$, Seiji Ito $^{2)}$, Toshihiro Miura ${ }^{3)}$ and Ken Horii ${ }^{1)}$ \\ 1) Faculty of Engineering, Kansai University \\ 2) Graduate School of Engineering, Kansai University \\ 3) Faculty of Letters, Kansai University
}

\begin{abstract}
This study investigated the relationship between the force applied to a finger and the differential threshold of the force. Further, it presented an improvement function for tactile perception in archers by adapting to circumstances in which enhanced tactile perception and finger dexterity are required to practice archery on a daily basis. For this purpose, a tactile display using an air jet was developed. The air was aimed at the center of the fingertip of the index finger. The inner diameter of the nozzle was set to $3 \mathrm{~mm}$. In this study, a psychophysical experiment was conducted to obtain the differential threshold from two subject groups - an archery athlete group and a control group. A total of six levels of standard stimuli ranging from $2.0 \mathrm{gf}$ to $7.0 \mathrm{gf}$ was obtained. As a result, the differential threshold of the archery group was significantly higher than that of the control group. The Weber ratio of the archery group remained around 0.13 and that of control group was 0.10 . The experiment also revealed that the differential threshold for archers exhibited less fluctuation between the trials and between the days, which implied that the tactile perception of archery athletes may be more stable than that of non-experienced subjects. This may be a plasticity property of tactile perception. J Physiol Anthropol 26(2): 143-148, 2007 http://www.jstage.jst.go.jp/browse/jpa2
\end{abstract}

[DOI: 10.2114/jpa2.26.143]

Keywords: Weber ratio, adaptation, differential threshold, tactile sensation, air-jet, archery athlete

\section{Introduction}

Mechanisms associated with human tactile sensation have not been researched as compared to other types of sensations such as visual and auditory sensations. In addition, a number of empirical studies on tactile sensation are largely dominated by studies on visual and auditory sensations (Maeno, 2004). On the other hand, perceptual characteristics of tactile sensation, including differential thresholds, have played a major role in technological applications such as virtual reality, health care, welfare, and entertainment (Ino et al., 1999 and Fujimoto et al., 2000). The study measuring the differential threshold originates from a study conducted by Weber, in which he used coins as tactile stimuli to measure the differential threshold and reported that the ratio of standard stimuli and the differential threshold was constant. This ratio is known as the Weber ratio (Weber, 1834/1978; Ifukube, 1997) investigated differential thresholds of tactile sensations more precisely than Weber, and reported that the Weber ratio remained constant until the standard stimulus remained as small as 100 gf. However, the Weber ratio only holds true at an intermediate stimuli level. Based on auditory and visual sensation studies, it was reported that the ratio is no longer constant when the stimulus is sufficiently small (Holway and Pratt, 1936). With regard to tactile sensation, Engen (1971) conducted a study on differential threshold for heaviness with a weight on one hand and reported that the Weber ratio increased as the stimulus - the weight on a hand - decreased.

The characteristics of the differential threshold of tactile sensation have been reported to provide very useful and effective information for the development of a virtual environment. Tactile display - one of the current applications using tactile-oriented virtual reality—requires a small dynamic force of an order of a few grams, at which the Weber ratio is no longer supposed to be constant (Baba et al., 2000; Amemiya, 1999). However, the fluctuation of the ratio when the tactile stimulus is as small as a few grams has not been investigated thus far (Ifukube, 1997).

In addition to the individual differences in the characteristics of the differential threshold of tactile sensation, an anthropological issue associated with adaptation to the circumstances in terms of the tactile differential threshold needs to be discussed for enhancing reality in a virtual environment. Fujimoto et al. (2000) showed that there were substantial variations in the correlation between standard 
stimuli and differential thresholds in an individual. With regard to the individual differences in human perception ability, the improvement in the perceptual function triggered by adaptation to the environment has been investigated by comparing sports athletes (i.e., well experienced) with non-athletes (i.e., inexperienced) (Seya and Mori, 2003). They showed the difference between these two groups using patterns of discrimination accuracy and concluded that sports athletes improved their perceptual abilities by adapting to sports practice on a daily basis. They reported that this phenomenon was not influenced only by the effectiveness of information processing based on their experience and knowledge, but also by perceptual plasticity under visually demanding circumstances.

Green and Bavelier (2003) measured the improvement in visual attention capacity using a similar research framework. They recruited subjects from two groups: frequent video-game players and non-video-game players and compared them empirically. As a result, they found that the frequent videogame players had an improved visual attention capacity and information processing ability, thereby showing conclusive evidence of the existence of the plasticity of human perception ability. To date, no study has directly exhibited such plasticity in tactile perception.

The objective of this study is twofold. First, the quantitative behavior of the Weber ratio is observed when a low level of tactile standard stimuli is provided. Secondly, the differences in the differential thresholds and Weber ratios of tactile sensation in the control subjects and the archers, who have adapted to the conditions in which enhanced tactile perception and finger dexterity are required to perform archery on a daily basis, are compared in order to discuss the existence of perceptual plasticity in tactile sensations.

\section{Methods}

\section{Subjects}

The subjects comprised individuals from two groups: archery athletes and control subjects. The subjects in the control group included six male university students aged from 19 to 21 years. In the archery group, there were eight male university students who belonged to the university archery team and whose experience in archery ranged between 1 and 3 years. The ages of the subjects in the archery group ranged from 22 to 24 years. The archery group has a training session for practicing archery on a daily basis. In the control group, the right hands of the subjects were used to measure the threshold, whereas, in the archery athlete group, the hands that pulled the bowstring were used for measurement.

\section{Apparatus}

For this experiment, a tactile display using an air jet was developed. The reason for choosing the air jet as a medium to generate the stimulus was to minimize the edge effect (Fransson-Hall and Kilbom, 1993), in which excessive tactile perception, which biases the tactile threshold, may be generated by the edge of the object if a solid-based medium, such as a pin-based display, is used to produce stimuli.

The system configuration of the air jet tactile display is shown in Figure 1. An air compressor (Hitachi Koki, $\mathrm{EC} 1430 \mathrm{H} 2)$ generated compressed air and the amount of air was controlled by a digital electro-pneumatic regulator (CKD, EVD-1900-P08 SN). The air jet was then produced by the operation of the solenoid valve (CKD, FAB11-M5-1-12C-3). The system was capable of producing an air jet of up to $12 \mathrm{gf}$ of applied force. The resolution of the force of the system was 0.1 gf.

The air was aimed at the center of the tip of the index finger. A clay base was used to immobilize the finger. The distal part

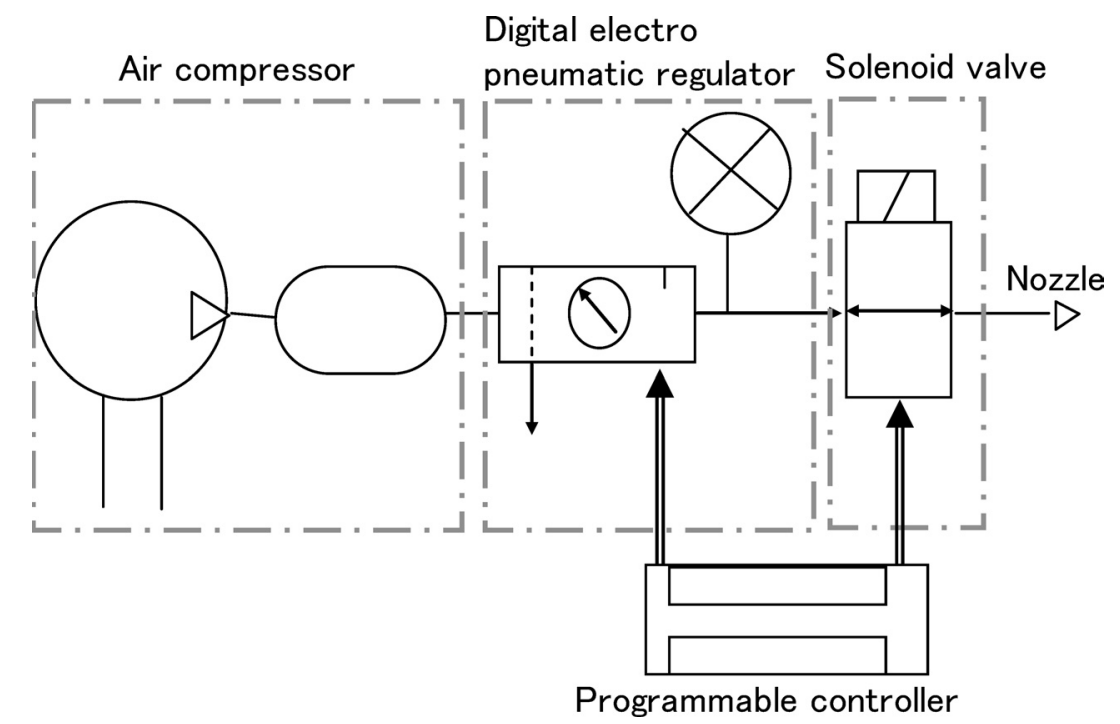

Fig. 1 Schematic diagram of air-jet-based tactile display 


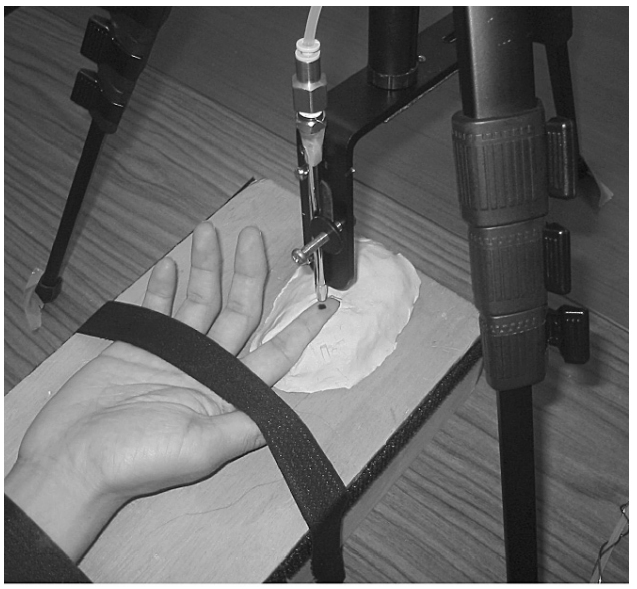

Fig. 2 Applying air-jet stimuli to the fingertip

of the palm and the wrist of the subjects were fixed tightly with Velcro fastening straps, as shown in Figure 2. The distance between the head of the nozzle and the surface of the fingertip was set to $5 \mathrm{~mm}$. Due to this, the fluctuation of the pressure of the air-jet was minimized, according to the pilot study. The inner diameter of the nozzle was set to $3 \mathrm{~mm}$, which was the typical size used in the study for investigating the application of the tactile display (for example, Asamura et al., 1998; Zappe et al., 2004). Throughout the trials, an eye mask was used in order to eliminate visual information associated with the intensity of the force, which is mainly visualized as tissue deformation due to the pressure. Earplugs were provided and the ears of the subjects were covered with headphones to counter any loud noise, including the sound generated by the air jet. This was done since there was a possibility of the subjects estimating the intensity of the force by listening to the pulse sound generated by the air jet emission.

\section{Stimulus}

In archery, the forces applied to the finger vary according to the process used: A compressive force is applied to the surface of the fingertip when the bow is drawn, whereas a sheer force is applied to the fingertip at the moment when the arrow is released. In our study, the compressive force was used as the stimulus.

A total of six levels of standard stimuli were provided, ranging from $2.0 \mathrm{gf}$ to $7.0 \mathrm{gf}$, in steps of $1.0 \mathrm{gf}$. A total of nine levels of comparison stimulus were provided, including four levels for stimuli higher than the normal standard, four levels for stimuli lower than the standard stimuli, and one level for the stimuli equal to the standard stimuli. We adopted a force level of $2.0 \mathrm{gf}$ to $7.0 \mathrm{gf}$ as the standard stimuli for the following reasons: (1) The range of the force adopted has been popularly used as the force intensity for tactile display, (2) Thus far, no studies have either examined such small force levels or shown the changing point of force as a standard stimulus such that the Weber ratio is no longer constant (Greenspan and Bolanowski, 1996; Holway and Pratt, 1936), and (3) Although archery athletes work with a considerably higher force while drawing a bow, it has been reported that the Weber ratio is independent of the magnitude of standard stimuli (Stevens and Green, 1996).

\section{Procedure}

The method of complete series, a psychophysical technique, was used to measure the differential threshold. The method belongs to the methods of constant stimuli; however, while most of these methods require prolonged time periods to perform, the one adopted does not require such extensive time periods (Mochizuki et al., 2003).

The stimulus presentation comprised a pair of stimuli, one of which was a standard stimulus while the other was a stimulus selected from nine levels of comparison stimuli. The participants were informed of the start of the process with a single beep provided through their headphones, followed by the first tactile stimulus with a duration of one second. There was a three-second interval without any stimulus after the first tactile stimulus; then the second stimulus was given again for one second. After the second stimulus, a 10-second no stimulus interval was provided and the system prompted the subjects to state if the second stimulus was stronger/weaker/equal or if they were unsure of the first stimulus. The order of the stimulus presentation was fully randomized. A total of 90 trials, 10 trials for each stimulus comparison, were performed. After every 20 trials, a short break was given for fatigue recovery. The differential threshold was calculated by using the following three equations according to the definition derived by Tanaka (1977).

Upper Lumen: $\quad S_{U}=\left(C_{U}+\frac{D}{2}\right)-\frac{\sum_{i=1}^{p} U_{i}}{n} D$

Lower Lumen:

$$
S_{L}=\left(C_{L}+\frac{D}{2}\right)+\frac{\sum_{i=1}^{p} L_{i}}{n} D
$$

Differential Threshold: $\quad \Delta L=\frac{S_{U}-S_{L}}{2}$

$\left(\begin{array}{l}C_{U}: \text { Maximum stimulus compared, } C_{L}: \text { Minimum } \\ \text { stimulus compared, } U_{i}: \text { Total number of judgments } \\ \text { that belong to the high lumen in the " } i \text { "th stimulus } \\ \text { comparison, } L_{i}: \text { Total number of judgments that } \\ \text { belong to the "low" lumen in the " } i \text { "th stimulus } \\ \text { comparison, } n \text { : Number of trials performed in each } \\ \text { stimulus comparison, } D: \text { Step size of comparison } \\ \text { stimulus, } p \text { : Total number of comparison stimulus }\end{array}\right)$

Hardness of the skin was measured after the trials were completed. A durometer (Type ESE, Elastron Inc.) was used in this measurement. The skin hardness did not have a unit due to 
its relative nature.

\section{Results}

Figure 3 shows the relationship between standard stimuli and differential thresholds in the two subject groups. Each plot shows the mean threshold over the trials with the standard error. In the figure, the differential thresholds of the archery group appeared to be higher than those for the control group. The average differential threshold of the archery athlete group was 0.63 , which was significantly higher than that of the control group $(0.50, t(82)=2.89, \mathrm{p}<.01)$. The result of the difference in the thresholds of the two groups implied that the tactile perception of the archery athlete group was less sensitive than that of the control group.

It has been reported that a proportional relationship exists between the standard stimuli and differential threshold when sensory input from different modalities is used for many types of perception (Stevens and Green, 1996). As shown in Figure 3 , the threshold of the archery group obtained in the experiment was increased relative to the increase in the standard stimulus, whereas the threshold for the control group exhibited fluctuating trends. The correlation coefficient was 0.93 for the archery athlete group and 0.40 for the control group.

Figure 4 shows the changes in the Weber ratio due to the standard stimulus. Each plot shows the mean threshold over trials with the standard error. Similar to the trends shown in Figure 3, the Weber ratios were higher for the archery group than for the control group. The average Weber ratio of the archery group was 0.15 , which was significantly higher than that for the control group $(0.12, t(82)=2.13, p<.05)$. The Weber ratio for the archery group remained around 0.13 when the standard stimulus was more than $5 \mathrm{gf}$ and gradually increased as the standard stimulus decreased. On the other hand, the Weber ratio for the control group persisted at around 0.10 when the standard stimulus exceeded $4 \mathrm{gf}$ and it gradually increased as the standard stimulus decreased.

Figure 5 shows the relationship between skin hardness and average differential threshold for each subject. As shown in the

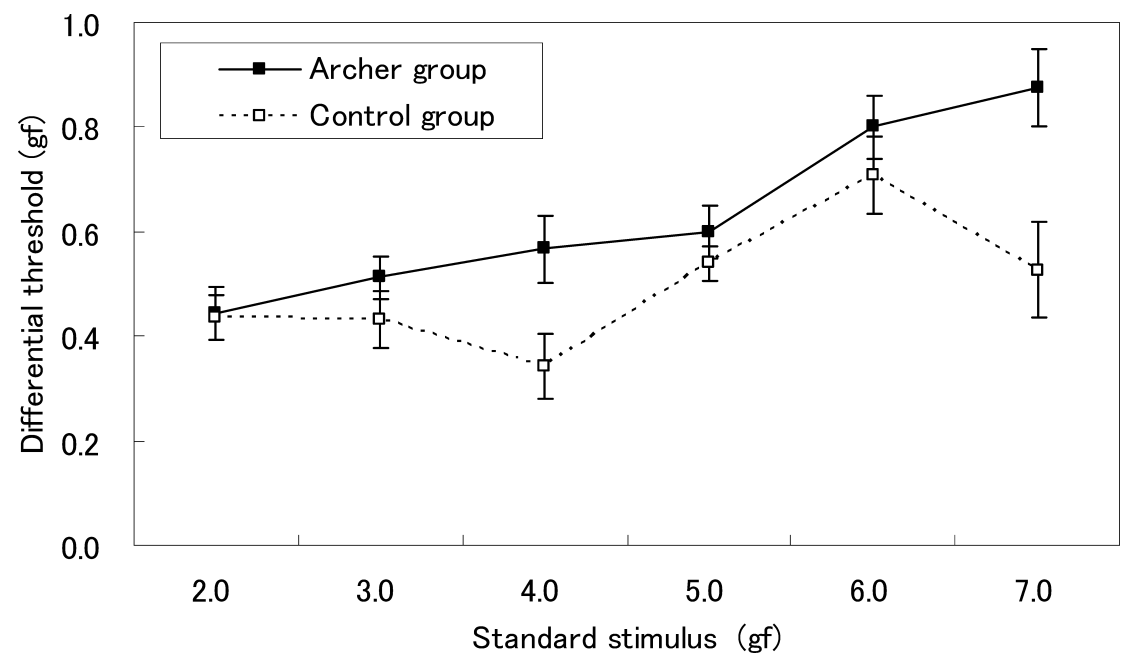

Fig. 3 Relationship between standard stimuli and differential threshold

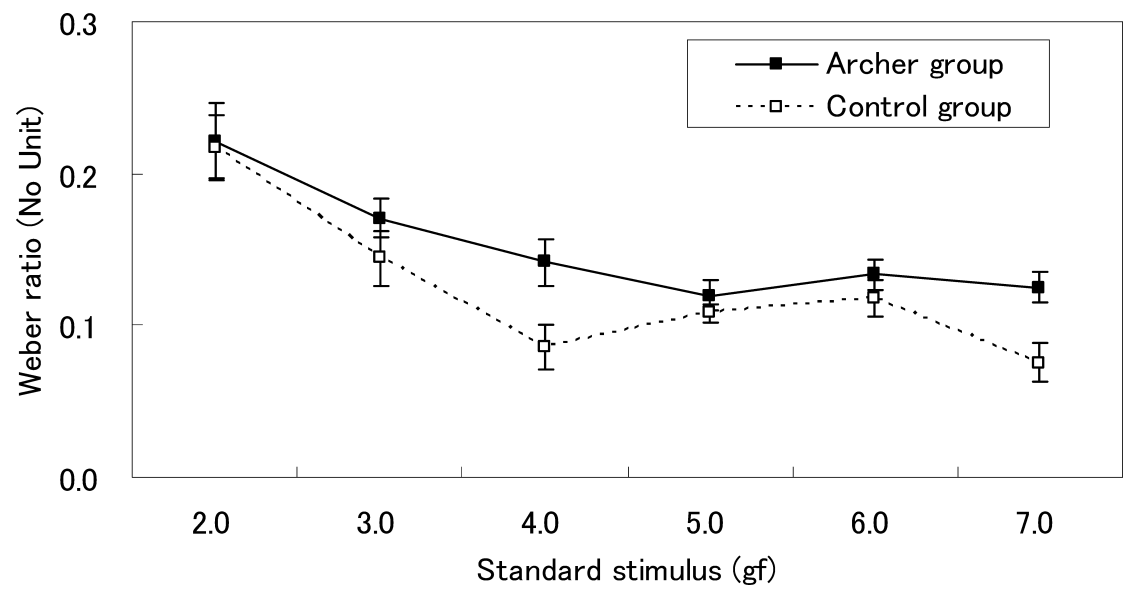

Fig. 4 Changes in Weber ratio by standard stimulus 


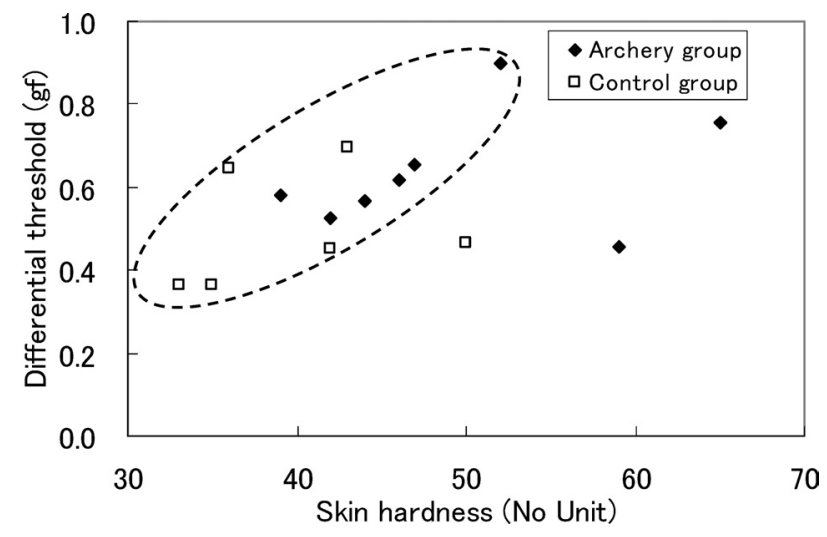

Fig. 5 Relationship between skin hardness and average differential threshold

figure, there was a tendency for the differential threshold to increase with skin hardness. By comparing the differences of the skin hardness between the two groups, the skin hardness of the archery athlete group (49.3) was significantly greater than that of the control group $(39.8, t(12)=2.199, p<.05)$.

\section{Discussion}

Few studies have obtained Weber ratios for tactile perception. Ifukube (1997) measured differential thresholds of the force that ranged from $100 \mathrm{gf}$ and $650 \mathrm{gf}$. They reported that the Weber ratio was 0.1 constantly over the ranges investigated. The Weber ratios obtained in our experiment were 0.13 for the archery group when the standard stimulus exceeded $5.0 \mathrm{gf}$, and 0.10 for the control group when the standard stimulus was more than $4.0 \mathrm{gf}$. The result obtained from the control group was consistent with the study by Ifukube (1997); however, the result obtained from the archery athlete group was slightly higher, i.e., the tactile sensitivity of the archers appeared to be less than that of the control subjects.

Our initial hypothesis was that archery athletes have a low differential threshold, i.e., their ability to feel and differentiate small forces is trained since they are accustomed to using their fingers to control force in order to aim at targets. According to the results of the Weber ratios obtained from the two groups, however, the hypothesis was observed to be incorrect. One explanation for this discrepancy between the hypothesis and the results may be the difference in the range of the standard stimuli used for the experiment and an ordinary archery performance. The average force required for drawing a bow is about 15-20 kgf (All Japan Archery Federation, 1988); thus, considerable force is applied to the fingertip. On the other hand, our experimental condition included standard stimuli ranging from $2.0 \mathrm{gf}$ to $7.0 \mathrm{gf}$, which appear to be much smaller than the force that an archer typically deals with. Although, as mentioned in the method section, the Weber ratio is independent of the magnitude of the standard stimulus (Greenspan and Bolanowski, 1996), archery athletes may have been unprepared for such small stimuli.
The first reason can explain why the Weber ratio of the archery athlete group was not lower than that of the control group; however, it cannot explain why the Weber ratio of the archery group was higher than that of the control group. The second reason is the contribution of skin hardness. Maeno et al. (1998) reported that tactile receptors detect skin deformation so that skin hardness may contribute to the level of sensitivity by changing the skin deformation of the finger indirectly. The results of our experiment showed that skin hardness had a strong and significant positive correlation with the differential threshold, which implies the possible contribution of skin hardness to masking tactile sensitivity. This yielded a high differential threshold. Next, the skin hardness of the archery group was higher than that of the control group; this may be attributed to an adaptation by practicing archery continually. Therefore, adaptation through archery practice, which requires fine force control using a finger, may not affect the sensitivity of the tactile perception of the finger but the skin hardness of the finger. This adaptation may reinforce the reason for the high Weber ratio, i.e., low tactile sensitivity, observed among the archery group.

Reproducibility of the threshold between the archery group and the control group also appears to be different. As the standard stimulus increases, the differential threshold for the archery group became relatively constantly higher, with a coefficient of determination of 0.93 . On the other hand, the differential threshold of the control group fluctuated with the decrease in the standard stimuli, with the coefficient of determination being 0.40 . Since the measurement of a set of trials for each standard stimulus required a day, based on the consistency of the standard stimuli, observed in the archery group, it can be interpreted that sensitivity in the archery group was more stable against between-day variability than that observed in the control group.

As a conclusion, we investigated the association between the force applied to the finger and the differential threshold of the force, and clarified the improvement function for tactile perception of the archers by adapting to the circumstances in which enhanced tactile perception and finger dexterity are required to practice archery on a daily basis. The study yielded the following results: The differential threshold of the archery group was not superior to that of the control group. Because the skin hardness of the archery group was significantly higher than that of the control group, it was inferred that the skin hardness may have produced a negative effect to improve the differential threshold. The experiment also revealed that the differential threshold for the archery athlete group showed very low fluctuations between the trials and between days. This implied that the tactile perception of archery athletes may be more stable than that of the non-experienced subjects, maybe as a plasticity property for tactile perception.

Acknowledgements This work was supported by JSPS KAKENHI (17570200). 


\section{References}

All Japan Archery Federation (1988) Archery textbook, Kodansha [In Japanese]

Amemiya K, Tanaka Y (1999) Portable tactile feedback interface using air jet. The 9th International Conference on Artificial Reality and Telexistence Proceedings 99: 115-122

Asamura N, Yokoyama N, Shinoda H (1998) Selectively stimulating skin receptors for tactile display. IEEE Computer Graphics and Applications 18, 6: 32-37

Baba S, Suga M, Oshiro O, Minato K, Matsuda T, Komori M, Takahashi T, Tokutomi Y (2000) The measurement of tactile sensation for the haptic device. Technical Report of IEICE 100, 350: 23-27 [In Japanese]

Engen T (1971) Psychophysics: discrimination and detection. In Kling JW, Riggs LA eds. Woodworth and Schlosberg's experimental psychology (3rd Ed.). New York: Holt

Fransson-Hall C, Kilbom $\AA$ (1993) Sensitivity of the hand to surface pressure. Appl Ergon 24, 3: 181-189

Fujimoto H, Yamakawa S, Manabe S (2000) A measurement of differential limen for constant force and elastic force of finger. J Hum Interface Society 2, 4: 67-72 [In Japanese]

Green CS, Bavelier D (2003) Action video game modifies visual selective attention. Nature 423: 534-537

Greenspan JD, Bolanowski SJ (1996) The psychophysics of tactile perception and its peripheral physiological basis. In Kruger L. Pain and Touch: Handbook of Perception and Cognition (2nd Ed.). Academic Press

Holway AH, Pratt CC (1936) The Weber ratio for intensitive discrimination. Psychol Rev 43: 322-340

Ifukube T (1997) A study on tele-existence of the tactile sense. J Robotics and Mechatronics 9, 3: 197-202

Ino S, Izumi T, Ifukube T (1999) Design of Haptic Interface System Based on Sensory Characteristics of Human Hand. J Hum Interface Society 1, 4: 9-17 [In Japanese]

Maeno T, Kobayashi K, Yamazaki N (1998) Relationship between the structure of human finger tissue and the location of tactile receptors. Bull JSME Int J 41, 1: 94-100

Maeno T (2004) Digital human and virtual reality: Tactile modeling and finite element analysis. Transactions of the Virtual Reality Society of Japan 9, 2: 72-77 [In Japanese]

Mochizuki K, Yamazaki S, Ohnishi H, Nakamura N, Yuki K (2003) The psychophysical evaluation of force feedback device. Technical Report of IEICE 103, 445: 107-110 [In Japanese]

Seya Y, Mori S (2003) Characteristic of reaction time during pursuit eye movement in karate. Technical Report of IEICE 103-522: 7-11 [In Japanese]

Stevens JC, Green BG (1996) History of research on touch. In Kruger L. Pain and touch: Handbook of Perception and Cognition (2nd Ed.). Academic Press

Tanaka Y (1977) Shinrigaku Sokuteiho, University of Tokyo Press [In Japanese]

Weber EH (1834/1978) De subtilitate tactu. In Ross HE, Murray DJ (Trans.), The sense of Touch. Academic Press

Zappe AC, Maucher T, Meier K, Scheiber C (2004) Evaluation of a pneumatically driven tactile stimulator device for vision substitution during fMRI studies. Magn Reson Med 51: $828-834$

This article was presented at the 8th International Congress of Physiological Anthropology, 2006 (ICPA 2006), in Kamakura, Japan

Received: September 28, 2006

Accepted: November 1, 2006

Correspondence to: Kentaro Kotani, Faculty of Engineering, Kansai University, 3-3-35, Yamate-cho, Suita, Osaka 5648680, Japan

Phone: 06-6368-1121

Fax: 06-6330-3154

e-mail: kotani@iecs.kansai-u.ac.jp 